\title{
Planejamento estatístico de experimentos aplicado ao desenvolvimento de formulações para revestimentos cerâmicos
}

\section{(Statistical design of experiments applied to the development of formulations for ceramic tiles)}

\author{
R. T. Zauberas ${ }^{1}$, P. L. S. Gomes ${ }^{2}$, C. A. R. Diniz', A. O. Boschi \\ ${ }^{1}$ LaRC, Departamento de Engenharia de Materiais - DEMa \\ ${ }^{2}$ Departamento de Estatística \\ Universidade Federal de $S$. Carlos \\ Rod. Washington Luiz, km 235, C. P. 676, S. Carlos, SP, 13565-905 \\ pzuba@iris.ufscar.br
}

\begin{abstract}
Resumo
Este trabalho avalia a utilização de técnicas de planejamento estatístico de experimentos com misturas envolvendo variáveis de processo no estudo de formulações para a produção de revestimentos cerâmicos, buscando minimizar o caráter empírico encontrado industrialmente nesta etapa do processamento. Misturas de três matérias-primas (argila, feldspato e areia), utilizadas industrialmente para a produção de revestimentos, foram ensaiadas sob condições padronizadas visando à quantificação da influência de cada matériaprima na resistência mecânica e na absorção de água das peças após a queima. As influências de duas variáveis de processo, pressão de compactação e temperatura de queima, também foram avaliadas. Os resultados obtidos demonstram o potencial de utilização das técnicas de planejamento estatístico de experimentos no estudo e desenvolvimento de formulações para revestimentos cerâmicos. Palavras-chave: revestimentos cerâmicos, planejamento estatístico de experimentos, variáveis de mistura, variáveis de processo.
\end{abstract}

Abstract

This work evaluates the use of statistical design of experiment on the development of formulations for ceramic tiles production, trying to minimise the empirical character industrially found on this processing step. Mixtures of three raw materials used in tile production (clay, feldspar and quartz sand), were processed under standardised conditions, aiming at the quantification of the contribution of each raw material influence on mechanical strength and water absorption of the bodies after sintering. The influences of two process variables, pressing and sintering temperature, were also evaluated. Results obtained show the potential on utilising statistical design of experiments with mixtures and process variables techniques on the study and development of formulations for ceramic tiles. Keywords: ceramic tiles, statistical design of experiments, mixture variables, process variables.

\section{INTRODUÇ̃̃O}

\section{Revestimentos cerâmicos}

Quando um produto é fabricado, deve-se ter em mente quais serão as propriedades finais que este deve possuir para desempenhar suas funções. Estas propriedades são determinadas pela microestrutura do material, cuja formação é dependente das matérias-primas e do processamento a que estas foram submetidas até a obtenção do produto acabado.

O processamento de revestimentos cerâmicos, que são produtos utilizados para revestir pisos, paredes e fachadas, inicia-se com a definição de uma massa ou formulação cerâmica, constituída de uma mistura de matérias-primas. Esta mistura, quando adequadamente processada, deve permitir a obtenção de um produto final com as características desejadas, capaz de desempenhar as funções para a qual foi projetado. Entretanto, a definição da massa ainda é a etapa do processamento de revestimentos que mais depende do conhecimento pessoal de alguns poucos indivíduos em uma fábrica, conhecimento este geralmente adquirido pela prática mas com base na tentativa e erro.

Os revestimentos cerâmicos são classificados de acordo com a adequação ao local de aplicação do produto. Revestimentos para pisos devem possuir baixa porosidade, apresentando elevada resistência mecânica e baixa absorção de água, ao passo que revestimentos para parede ou azulejos necessitam de alta 
porosidade para garantir baixa retração de queima, alta estabilidade dimensional e facilidade de instalação. Na Tabela I é apresentada a classificação dos revestimentos cerâmicos prensados [1], além da absorção de água e resistência mecânica exigidas por norma [2].

Tabela I: Classificação de revestimentos cerâmicos produzidos por prensagem

[Table I: Pressed ceramic tiles classification.]

\begin{tabular}{lccccc}
\hline Característica & \multicolumn{5}{c}{ Classificação } \\
\hline & BI & BIb & BIIa & BIIb & BIII \\
\hline Absorção & AA & $0,5<$ AA & $3<$ AA & $6<$ AA & AA \\
de água & $\leq 0,5$ & $\leq 3$ & $\leq 6$ & $\leq 10$ & $>10$ \\
$(\%)$ & & & & & \\
\hline $\begin{array}{l}\text { Módulo de } \\
\text { resistência }\end{array}$ & MRF & MRF & MRF & MRF & MRF \\
à flexão & & $\geq 30$ & $\geq 22$ & $\geq 18$ & $\geq 15$ \\
$(\mathrm{MPa})$ & & & & & \\
\hline
\end{tabular}

Métodos estatísticos em experimentos com misturas

Em sistemas envolvendo misturas de diferentes constituintes, onde a variação do teor de uma matéria-prima não pode ser realizada sem a alteração dos teores das outras, a técnica estatística mais adequada é o planejamento de experimentos com misturas [3].

Um experimento com mistura é um experimento cuja variável de resposta (característica de interesse) é função somente das proporções relativas dos ingredientes presentes na mistura e não da quantidade total da mistura. As proporções dos diversos componentes de uma mistura não são independentes; elas devem ser não negativas, equação (A), e se forem expressas como frações da mistura, a soma das proporções deve ser igual a 1 , equação (B). Neste trabalho tratamos de um experimento com mistura envolvendo três componentes $(\mathrm{q}=3): \mathrm{x}_{1}(\operatorname{argila}), \mathrm{x}_{2}\left(\right.$ feldspato) e $\mathrm{x}_{3}$ (areia).

$$
\begin{array}{r}
\mathrm{x}_{i} \geq 0 \quad i=1, \ldots, \mathrm{q} \\
\sum_{\mathrm{i}=1}^{\mathrm{q}} \mathrm{x}_{i}=1
\end{array}
$$

Além das restrições naturais mostradas nas equações (A) e (B), pode-se também ter restrições superiores e inferiores dos teores dos componentes na mistura, por motivos tecnológicos ou econômicos, entre outros. Tais restrições são escritas conforme a equação (C).

$$
0 \leq \mathrm{L}_{\mathrm{i}} \leq \mathrm{x}_{\mathrm{i}} \leq \mathrm{U}_{\mathrm{i}} \leq 10 \quad i=1, . ., \mathrm{q}
$$

onde algum $L_{\mathrm{i}}$ pode ser zero e algum $\mathrm{U}_{\mathrm{i}}$ pode ser um, indicando que o componente está livre de limitações.

A região de factibilidade de misturas definida pelas equações (A), (B), e (C) é um hiperpoliedro convexo. Neste estudo, as restrições definidas inicialmente sobre as quantidades das

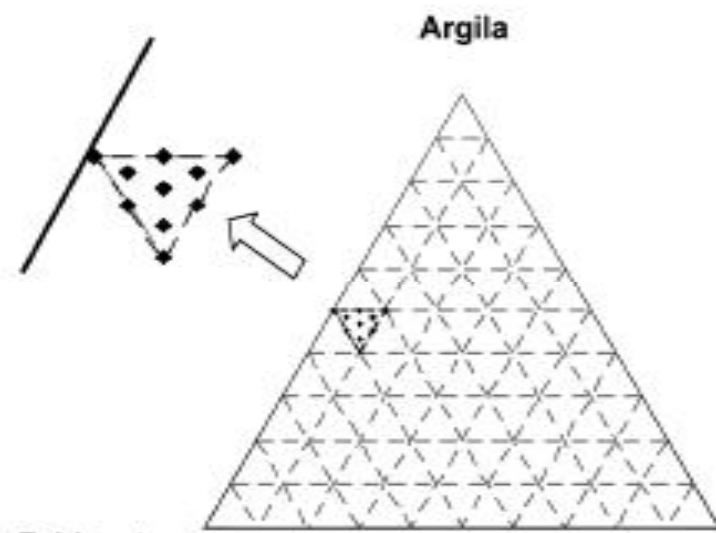

Feldspato

Areia

Figura 1: Sub-região do simplexo redefinida para $0,4 \leq x_{1} \leq 0,5$, $0,4 \leq \mathrm{x}_{2} \leq 0,5$ e $0 \leq \mathrm{x}_{3} \leq 0,1$.

[Figure 1: Simplex sub-region redefined to $0.4 \leq x_{1} \leq 0.5,0.4 \leq x_{2} \leq 0.5$ e $0 \leq x_{3} \leq 0.1$.]

matérias-primas foram $0,35 \leq x_{1} \quad 0,50,0,35 \leq x_{2} \leq 0,50$ e $0 \leq \mathrm{x}_{3} \leq 0,10$. Através da análise de factibilidade dos limites superiores e inferiores das matérias-primas, estes foram alterados para os limites apresentados na Fig. 1.

Inclusão de variáveis de processo em experimentos com misturas

Além das proporções de mistura, existem outras variáveis chamadas variáveis de processo que representam condições externas cujos níveis, quando alterados, podem afetar as propriedades dos componentes da mistura. Neste experimento, as variáveis de processo avaliadas foram a pressão de compactação e a temperatura de queima, codificadas como $z_{1}$ e $z_{2}$.

Em experimentos com misturas envolvendo variáveis de processo, o delineamento experimental é estabelecido através de uma combinação do delineamento associado às variáveis de mistura com um delineamento associado às variáveis de processo, estabelecendo-se um delineamento de misturas para cada combinação dos níveis das variáveis de processo. Na definição do experimento contendo componentes de misturas e variáveis de processo, temos que as regiões de interesse das variáveis de processo e dos componentes de misturas são um hiper cubo ndimensional [4] e um simplexo (q -1) dimensional [5], respectivamente. Neste experimento, como $n=2$ temos um quadrado e como $\mathrm{q}=3$ temos um triângulo eqüilátero (sem considerar as restrições impostas pelos limites das matériasprimas). A região de interesse combinada pelos componentes de misturas e pelas variáveis de processo tem dimensão $q-1+n$.

Foi escolhido para este estudo um delineamento associado às variáveis de mistura conhecido como "Gerado por Computador" [6], capaz de ajustar e verificar a adeqüabilidade de um modelo de superfície de resposta até ordem 3, conforme a equação (D). O delineamento associado às variáveis de processo é expresso na equação (E). Os coeficientes $\beta_{1}, \beta_{2} \mathrm{e}$ $\beta_{3}$ e $\alpha_{1}$ e $\alpha_{2}$ são associados, respectivamente, às contribuições das matérias-primas e das variáveis de processo nas variáveis 


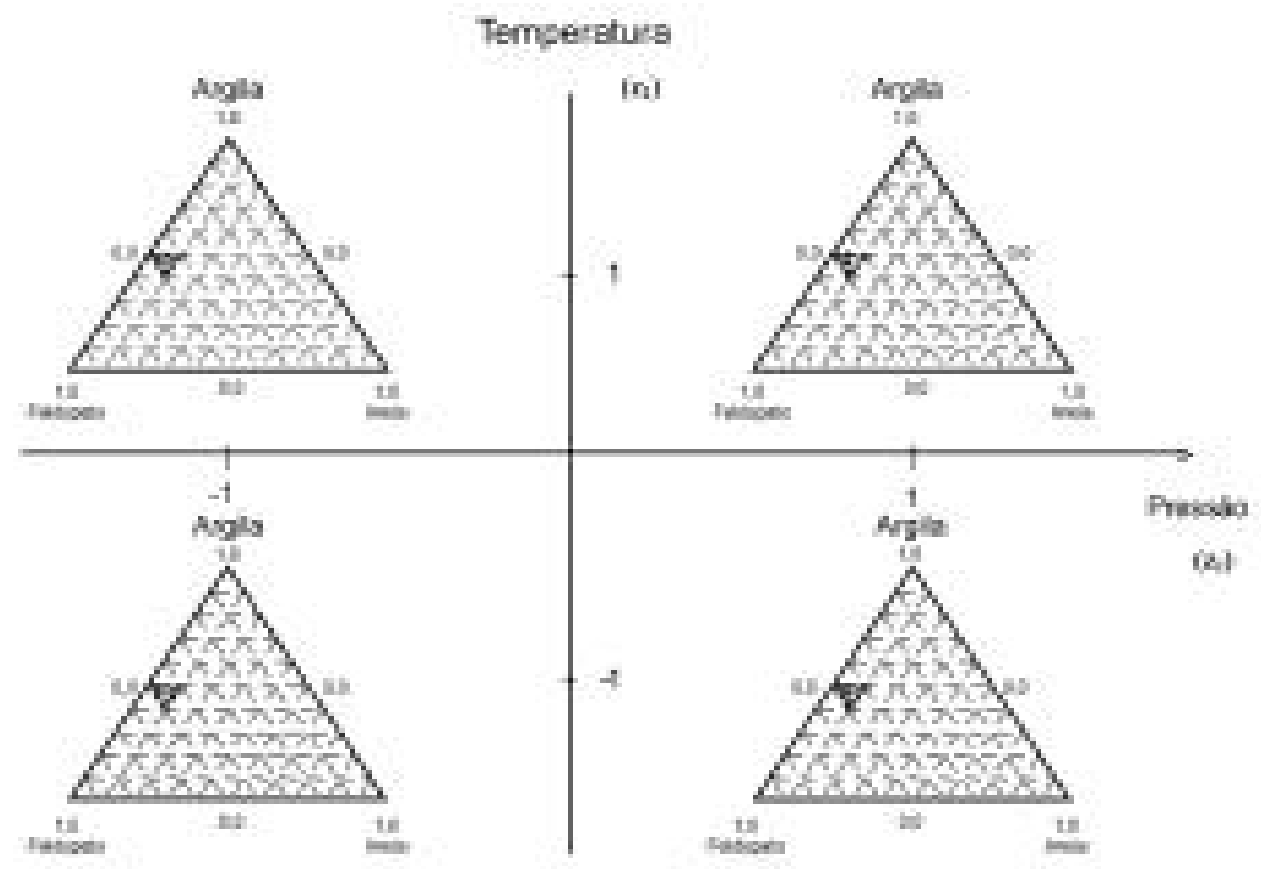

Figura 2: Delineamento combinado entre delineamento de misturas $(\mathrm{q}=3)$ e delineamento fatorial $2^{2}$.

[Figure 2: Mixed design of mixtures design $(q=3)$ and 22 factorial design.]

de resposta, sendo os termos $\varepsilon_{\mathrm{m}}$ e $\varepsilon_{\mathrm{p}}$ os erros aleatórios associados a cada delineamento.

$$
\begin{aligned}
& \mathrm{y}_{\mathrm{m}}=\beta_{1} \mathrm{x}_{1}+\beta_{2} \mathrm{x}_{2}+\beta_{3} \mathrm{x}_{3}+\varepsilon_{\mathrm{m}} \\
& \mathrm{y}_{\mathrm{p}}=\alpha_{0}+\alpha_{1} \mathrm{z}_{1}+\alpha_{2} \mathrm{z}_{2}+\varepsilon_{\mathrm{p}}
\end{aligned}
$$

Na Fig. 2 tem-se o delineamento gerado por computador combinado com o planejamento fatorial.

A partir da coleta dos pontos estabelecidos do delineamento da Fig. 2, tem-se o modelo global de superfície de resposta a ser ajustado, equação $(\mathrm{F})$.

$$
\begin{aligned}
& y=\{\text { modelo de misturas }\}\{\text { modelo fatorial }\} \\
& y=\left(\beta_{1} x_{1}+\beta_{2} x_{2}+\beta_{3} x_{3}\right)\left(\alpha_{0}+\alpha_{1} z_{1}+\alpha_{2} z_{2}\right)+\varepsilon \\
& y=\sum_{i=1}^{3}\left(\gamma_{i}^{0}+\sum_{k=1}^{2} \gamma_{i}^{v} z_{2}\right) x_{i}
\end{aligned}
$$

onde $\quad \gamma_{i}^{k}=\alpha_{k} \beta_{i}$

Esta metodologia compreende um grupo de técnicas estatísticas para construção e exploração de modelos empíricos. Através de um cuidadoso planejamento e análise de experimentos, ela busca relacionar uma resposta, ou variável de saída, aos níveis de um número de variáveis de entrada que a afetam; e também encontrar o "melhor" valor dessa resposta. Se descobrir o melhor valor ou se os valores da resposta estiverem além dos recursos disponíveis do experimento, então esta metodologia será usada para pelo menos se obter um melhor entendimento da resposta global do sistema.

\section{MATERIAIS E MÉTODOS}

As matérias-primas deste trabalho, uma argila, um feldspato e uma areia, foram escolhidas aleatoriamente entre diversas amostras de matérias-primas cerâmicas industriais disponíveis. A definição das formulações e a ordem de realização dos ensaios foram feitas através do software Minitab13, com as seguintes restrições iniciais quanto aos teores de cada matéria-prima: argila entre $35 \%$ e $50 \%$, feldspato entre $35 \%$ e $50 \%$ e areia entre $0 \%$ e $10 \%$. As variáveis de processo avaliadas foram a pressão de compactação $\left(375 \mathrm{kgf} / \mathrm{cm}^{2}\right.$, codificada como $\mathrm{z}_{1}=\mathrm{P}-1$ ou $458 \mathrm{kgf} / \mathrm{cm}^{2}$, codificada como $\left.\mathrm{z}_{1}=\mathrm{P}+1\right)$ e a temperatura de queima $\left(1175{ }^{\circ} \mathrm{C}\right.$, codificada como $\mathrm{z}_{2}=\mathrm{T}-1$, e $1225{ }^{\circ} \mathrm{C}$, codificada como $\mathrm{z}_{2}=\mathrm{T}+1$ ). $\mathrm{O}$ delineamento gerado por computador combinado com planejamento fatorial $2^{2}$ consistiu de um total de 48 ensaios avaliando dez diferentes formulações, sendo o delineamento de misturas estabelecido em cada um $\operatorname{dos} 2^{2}=4$ pontos do delineamento fatorial. Após a dosagem de cada formulação, foi realizada a moagem a úmido em moinho de bolas por $20 \mathrm{~min}$, tendo cada barbotina a mesma massa de água e de sólidos e $0,75 \%$ de silicato de sódio (base seca).

As barbotinas foram secas a $110{ }^{\circ} \mathrm{C}$, desagregadas, umidificadas com $8 \%$ de umidade, granuladas em malha de $500 \mu \mathrm{m}$ e armazenadas por um dia para homogeneização da umidade. Com o material granulado foram prensadas cinco peças para cada corrida do experimento (combinação das variáveis de mistura e variáveis de processo) em moldes de 
$6 \times 2 \mathrm{~cm}^{2}$, as quais foram secas e então queimadas. Os ciclos de queima foram realizados com taxa de $10^{\circ} \mathrm{C} / \mathrm{min}$ até $1000^{\circ} \mathrm{C}$, taxa de $7{ }^{\circ} \mathrm{C} / \mathrm{min}$ até a temperatura máxima e patamar nesta temperatura de $3 \mathrm{~min}$; o resfriamento foi natural, dentro do forno. A caracterização após a queima avaliou a resistência

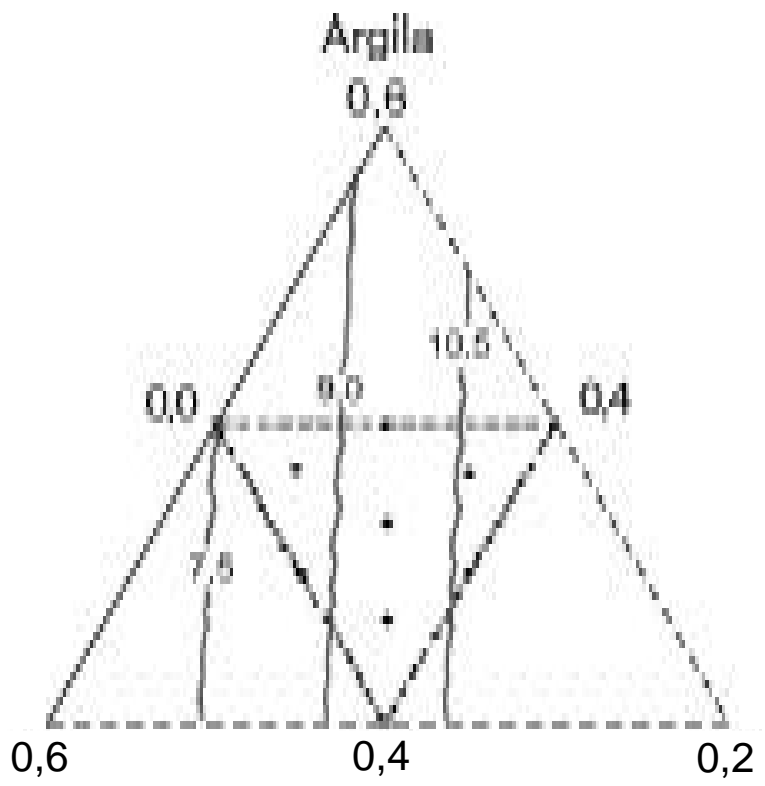

Feldspato

Areia

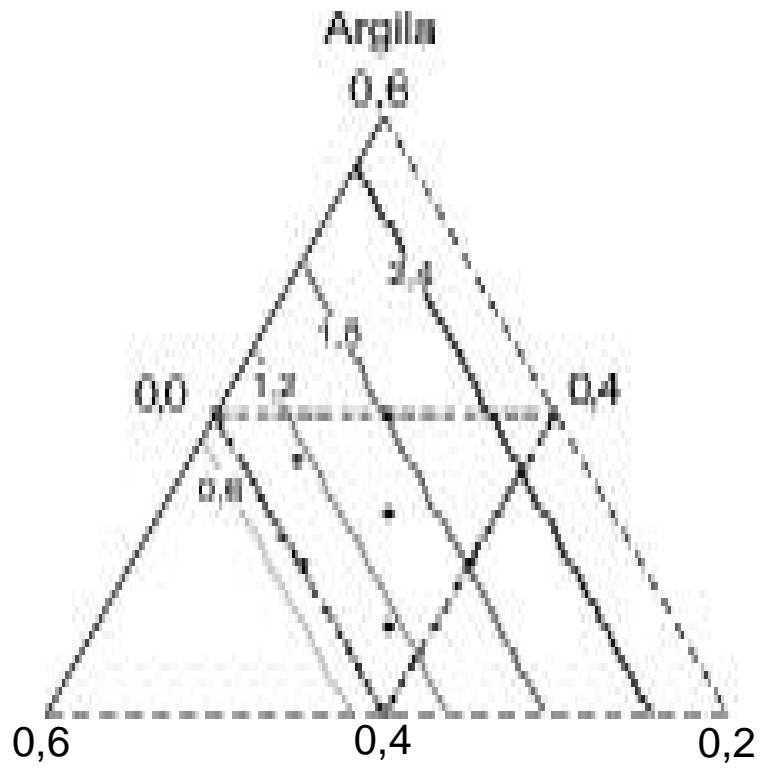

Feldspato

Areia

Figura 3: Superfície de resposta para a absorção de água nas queima realizadas a (a) $1175^{\circ} \mathrm{C}$ e (b) $1225^{\circ} \mathrm{C}$.

[Figure 3: Water absorption response surface of firings at (a) $1175^{\circ} \mathrm{C}$ and (b) $1225^{\circ} \mathrm{C}$.] mecânica por ensaio de flexão em três pontos e a absorção de água.

Após a execução do experimento, os resultados obtidos foram fornecidos ao software que por meio da ferramenta estatística DOE (Design of Experiments), processou as informações via técnica de Experimentos com Misturas Envolvendo Variáveis de Processo.

\section{RESULTADOS E DISCUSSÃO}

Os intervalos de variação dos teores de cada matéria-prima inicialmente definidos, após checagem de compatibilidade dos limites inferiores e superiores, foram redefinidos para argila entre $40 \%$ e $50 \%$, feldspato entre $40 \%$ e $50 \%$ e areia entre $0 \%$ e $10 \%$. O software forneceu 40 condições experimentais (dez formulações, cada uma processada nas condições P-1T-1, P$1 \mathrm{~T}+1, \mathrm{P}+1 \mathrm{~T}-1$ e $\mathrm{P}+1 \mathrm{~T}+1$ ), além de duas réplicas dos pontos centrais do delineamento de misturas, com um total de 48 ensaios. Os resultados da caracterização das peças produzidas pelo processamento das formulações ensaiadas foram fornecidos ao software para o tratamento estatístico dos dados.

A variável de resposta absorção de água teve um modelo ajustado de ordem um (equação de primeiro grau), com uma relação empírica aproximada altamente significativa $\left(\mathrm{F}_{\text {observado }}>>\mathrm{F}_{\text {crítico }}\right)$ e apresentando $\mathrm{R}^{2}$ de $96,7 \%$, (indicativo de quanto o modelo foi capaz de explicar a variabilidade existente nos dados observados). O resíduo foi normal e independentemente distribuído, com média zero e variabilidade constante. Os coeficientes relacionados às variáveis de processo indicam que a variável pressão de compactação, $\mathrm{z}_{1}$, não contribui significativamente $(\mathrm{p}>0,05)$ na absorção de água, sendo então esta variável desconsiderada na modelagem da superfície de resposta. Na equação (G) tem-se o modelo obtido, apresentando-se entre parênteses o erro associado a cada variável.

$\hat{\mathrm{y}}\left(\mathrm{x}_{1}, \mathrm{x}_{2}, \mathrm{x}_{3}, \mathrm{z}_{1}, \mathrm{z}_{2}\right)=\begin{gathered}12,22 \mathrm{x}_{1}-7,08 \mathrm{x}_{2}+24,87 \mathrm{x}_{3}-13,25 \mathrm{x}_{3} \mathrm{z}_{2} \\ (1,484)(1,484)\end{gathered}$

Através da análise dos coeficientes associados aos teores de cada matéria-prima e à temperatura de queima, nota-se que o feldspato $\left(\mathrm{x}_{2}\right)$ é a matéria-prima que mais contribui na redução da absorção de água; pode ser notado também um comportamento diferenciado da areia $\left(\mathrm{x}_{3}\right)$ em relação à temperatura, evidenciado pelo coeficiente de interação entre $\mathrm{x}_{3}$ e $\mathrm{z}_{2}$. Um possível evento relacionado a esta interação é o início da dissolução das partículas de areia com o aumento da temperatura de $1175^{\circ} \mathrm{C}$ para $1225^{\circ} \mathrm{C}$, devido à redução da viscosidade da fase vítrea formada. Na Fig. 3 são apresentadas as curvas de nível das superfícies de resposta do modelo para a absorção de água das peças queimadas a $1175^{\circ} \mathrm{C}$ e $1225^{\circ} \mathrm{C}$.

A variável de resposta resistência mecânica à flexão não teve um modelo ajustado capaz de explicar a variabilidade existente nos dados observados, mesmo através da utilização de um modelo de ordem três (cúbico especial), apresentando $\mathrm{F}_{\text {observado }} \sim 1,25 * \mathrm{~F}_{\text {crítico }}$ e $\mathrm{R}^{2}$ de $37,7 \%$. 


\section{CONCLUSÕES}

A técnica de planejamento de experimentos utilizada neste trabalho foi capaz de ajustar um modelo estatístico que explicou adequadamente a variabilidade da absorção de água em função das alterações dos teores das matériasprimas e das variáveis de processo. Entretanto, a técnica não permitiu ajustar um modelo para o módulo de resistência à flexão, sendo talvez necessária a adoção de alguma nova condição padronizada no processamento para a obtenção de um modelo capaz de descrever a variação desta característica de interesse.

\section{AGRADECIMENTOS}

O presente trabalho foi realizado com o apoio do Conselho Nacional de Desenvolvimento Científico e Tecnológico, CNPq.

\section{REFERÊNCIAS}

[1] Associação Brasileira De Normas Técnicas; Norma Técnica NBR 13817 Placas cerâmicas para revestimento - classificação (1997) 3 p.

[2] Associação Brasileira De Normas Técnicas; Norma Técnica NBR 13818 Placas cerâmicas para revestimento - especificação e métodos de ensaio (1997) $78 \mathrm{p}$.

[3] R. R. Villafranca, Diseño de experimentos para la optimización de mezclas, ATC (1999).

[4] G. E. P. Box, N. R. Draper, Empirical model-building and response surfaces, Wiley, New York (1987) 669p.

[5] J. A. Cornell, Experiments with mixtures, Wiley, New York, (1981) 305p.

[6] D. C. Montgomery, Design and Analysis of Experiments, 2nd Ed., John Wiley and Sons, New York (1984) 538 p.

(Rec. 05/07/03, Ac. 05/12/03) 